\title{
Population pharmacokinetics of mefloquine, administered as a fixed-dose combination of artesunate-mefloquine in Indian patients for the treatment of acute uncomplicated Plasmodium falciparum malaria
}

Vincent Jullien ${ }^{1,2}$, Neena Valecha ${ }^{3}$, Bina Srivastava ${ }^{3}$, Bhawna Sharma ${ }^{4}$ and Jean-René Kiechel $\left.\right|^{5^{*}}$

\begin{abstract}
Background: Fixed-dose combinations of artemisinin combination therapy are strongly recommended to facilitate drug administration and compliance. New fixed-dose combinations must nevertheless be evaluated in relevant populations in terms of efficacy and pharmacokinetics.

Methods: A single-arm, open-label, clinical trial was performed in Indian patients with acute uncomplicated Plasmodium falciparum malaria to investigate the efficacy and the pharmacokinetics of mefloquine when combined with artesunate in a fixed-dose combination (400/200 mg of mefloquine base/artesunate). The pharmacokinetic analysis was performed using a population approach.

Results: Seventy-seven patients were included in the study. Mefloquine pharmacokinetics obeys a two-compartment model with first-order absorption and elimination. Mean parameter estimates (\% inter-individual variability) were as follows: $0.16 \mathrm{~h}^{-1}$ (75\%) for the absorption rate constant, $1.13 \mathrm{~L} / \mathrm{h}$ (30\%) for the apparent plasma clearance, $271 \mathrm{~L}(21 \%)$ for the apparent central distribution volume, $344 \mathrm{~L}(54 \%)$ for the apparent peripheral distribution volume, and $1.43 \mathrm{~L} / \mathrm{h}$ for the apparent distribution clearance. These values were consistent with the pharmacokinetic results described in Thai patients. No significant covariate was found for clearance. Body weight explained the inter-individual variability of the apparent central and peripheral distribution volumes. The PCR-adjusted efficacy of the treatment was 100\%.

Conclusions: The lack of significant covariate explaining the inter-individual variability of mefloquine clearance, combined with the excellent efficacy, supports the use of the standard 200/400 mg of artesunate-mefloquine fixed-dose combination in Indian patients with uncomplicated $P$. falciparum malaria.

Trial Registration: Clinical Trial Registration: ISRCTN70618692

Keywords: Mefloquine, Population pharmacokinetics, Adults, Malaria
\end{abstract}

\footnotetext{
* Correspondence: jean-rene.kiechel@wanadoo.fr

${ }^{5}$ Drugs for Neglected Diseases initiative, 15 chemin Louis-Dunant, 1202

Geneva, Switzerland

Full list of author information is available at the end of the article
} 


\section{Background}

Artemisinin combination therapy (ACT) is now the treatment of choice for uncomplicated Plasmodium falciparum malaria [1,2]. These combinations involve a rapidly eliminated and fast-acting artemisinin derivative, responsible for a rapid decline in the parasite biomass, together with a much slower eliminated drug that kills the remaining parasites. Mefloquine (MQ), is one of the partner drugs that can be combined with an artemisinin derivative, with the artesunate (AS)/MQ combination shown to be effective in several clinical trials [3-5], and used extensively in countries across Southeast Asia, the Western Pacific, Africa and Latin America over the last two decades [6]. The World Health Organization (WHO) currently recommends five formulations of ACT for the treatment of uncomplicated $P$. falciparum malaria, including the AS and MQ combination [7].

Fixed-dose combinations (FDC) are now strongly recommended compared to loose tablets because fewer tablets are involved and patient adherence should be improved [8]. The FDC of AS/MQ has been demonstrated to be efficacious and safe for uncomplicated malaria treatment in studies carried out in Thailand [9], Myanmar [10], India and Cambodia [11], as well as in a large intervention study on 23,845 patients in Brazil [12]. It is nevertheless necessary to verify not only the efficacy but also the pharmacokinetics of compounds administered as a FDC in a target population, in order to investigate possible population-related differences due to metabolism or food-drug interactions.

The present study investigated the efficacy, tolerability and pharmacokinetics of the AS/MQ FDC in Indian adult patients with acute uncomplicated $P$. falciparum malaria in highly endemic areas. Despite previous reports of high degrees of resistance to chloroquine in five Indian states, no MQ resistance was found [13]. The efficacy and safety results have already been reported [14], and here the population pharmacokinetics of mefloquine are presented.

\section{Methods}

An open-label, single arm, multicentre study was carried out from December 2007 to November 2008 in patients of more than 18 years of age with $P$. falciparum malaria having an asexual parasitaemia density of between 1,000 and 100,000 parasites/ $\mu$ l of blood together with a fever $\geq 37.5^{\circ} \mathrm{C}$. Patients with signs of severe malaria, febrile conditions due to a disease other than malaria, a history of anti-malarial treatment in the previous 15 days, anaemia, hepatic or renal impairment, a history of cardiovascular, respiratory, gastrointestinal, neurological, malignancy, psychiatric, or endocrine disorders were excluded. Pregnant or lactating patients were also excluded. The study was conducted in accordance with the local laws and regulations, Indian Good Clinical Practices, Ethical guidelines on biomedical research issued by the Indian Council of Medical Research and the International Conference on Harmonisation-Good Clinical Practices. The protocol was reviewed and approved by the Ethics Committee of the National Institute of Malaria Research, New Delhi, Goa Medical College and Hospitals, Goa and Kasturba Medical College, Mangalore. Written consent from each of the subjects were obtained before enrolment in the study.

Subjects were orally administered two tablets of AS/MQ FDC, containing $100 \mathrm{mg}$ of AS and $200 \mathrm{mg}$ of MQ base per tablet, once daily and over three consecutive days. Blood samples for analysis of MQ plasma concentrations were collected before dosing on D0, D3 (72 hours after first dosing), D7, and on one other occasion at a randomly selected time on day 28,35 or 42 .

An automated liquid chromatography - tandem mass spectrometry method ( LC-MS/MS) for the quantification of AS, MQ and dihydroartemisinin (DHA) levels in human plasma samples was developed and validated. These compounds were extracted from human plasma using a solid phase extraction procedure and injected into the liquid chromatograph coupled to a tandem mass spectrometric detector, and quantified by use of the internal standard method.

MQ was quantified using the Multiple Reaction Monitoring (MRM) transitions of $379.20 \rightarrow$ 361.atomic mass units $(\mathrm{amu})$ and amodiaquine as an internal standard was quantified using MRM transitions of $356.70 \rightarrow 283.20$ amu. A weighted linear regression using weighting $1 /$ concentration $^{2}$ was prepared to determine the concentrations of AS, MQ and DHA in human plasma. Eight-point calibration curves were prepared (20.2 $\mathrm{ng} / \mathrm{mL}$ to $1,514.2 \mathrm{ng} / \mathrm{mL}$ for AS, $9.9 \mathrm{ng} / \mathrm{mL}$ to $3,455.5 \mathrm{ng} / \mathrm{mL}$ for $\mathrm{MQ}$, and $39.2 \mathrm{ng} / \mathrm{mL}$ to $2,940.0 \mathrm{ng} / \mathrm{mL}$ for DHA), and used to determine concentrations of AS, MQ and DHA in subject samples. The Lower Limit of Quantification for MQ (LLOQ) was $9.9 \mathrm{ng} / \mathrm{ml}$.

The bio-analytical method was validated for various validation parameters, such as specificity and selectivity, sensitivity, carry-over, solution linearity, precision and accuracy, recovery, stability, dilution integrity, matrix effect, re-injection reproducibility, and ruggedness.

During before study method validation for MQ the within batch precision (\% CV) ranged from $2.14 \%$ to $6.99 \%$ and the between-batch precision ranged from $3.83 \%$ to $0.83 \%$. The within batch accuracy (\% Nominal) ranged from $90.91 \%$ to $114.41 \%$ and the between-batch accuracy ranged from 99.32 to $104.04 \%$.

The between-run precision (\%CV) for the Quality Control Samples of MQ during the study was $6.49 \%$, $4.16 \%$ and $9.11 \%$ for LQC, MQC and HQC samples. The between-run accuracy (\% Nominal) for the quality Control 
Samples of MQ during the study was $98.15 \%, 96.97 \%$ and 98.87\% for the same samples respectively.

Concentration-time data were analysed by use of the first-order conditional estimation method with interaction of the non-linear mixed effects modelling program NONMEM (version VI, version 2.0, double precision) $[13,15]$. Three structural PK models (one, two, and three compartment models), with first-order absorption and first-order elimination from the central compartment were investigated. The estimated pharmacokinetic parameters were the absorption rate constant $(\mathrm{ka})$, the apparent elimination clearance (CL/F, F being the bioavailability), the apparent central distribution volume $(\mathrm{Vc} / \mathrm{F})$ and, if relevant, apparent peripheral distribution volumes $\left(\mathrm{Vp} / \mathrm{F}\right.$ for a 2-compartment model, $\mathrm{Vp}_{1} / \mathrm{F}$ and $\mathrm{Vp}_{2} \mathrm{~F}$ for a 3-compartment model) and distribution clearances $\left(\mathrm{Q} / \mathrm{F}\right.$ for a 2-compartment model, $\mathrm{Q}_{1} / \mathrm{F}$ and $\mathrm{Q}_{2} / \mathrm{F}$ for a 3-compartment model). Three different residual errors models (additive, proportional, and combined additive-proportionnal) were tested to investigate the residual variability. Interindividual variability was described by an exponential model, assuming that individual parameters arise from a multivariate lognormal distribution with mean vector and variance-covariance matrix to be estimated. The \% interindividual variability was therefore calculated as the root mean square of the omega ${ }^{2}$ value given by NONMEM. Systematic testing for the influence of continuous covariates on the pharmacokinetic parameters was done by use of a generalized allometric model, according to the following equation, by using, for example, $C L / F$ and $B W$ :

$$
\frac{C L}{F}=T V\left({ }^{C L} / F\right) \times\left(\frac{B W}{\operatorname{median} B W}\right)^{\theta}
$$

where $T V(C L / F)$ was the typical value of the apparent clearance for a patient with the median covariate value, and $\theta$ was the influential factor for body weight (BW).

Continuous tested covariates for clearance were age, bodyweight, parasitaemia, hepatic enzymes, haemoglobin, and serum creatinin. Continuous covariates tested for distribution volume were bodyweight, haemoglobin, and parasitaemia.

The possible influence of the day of administration on $\mathrm{CL} / \mathrm{F}$ was investigated as follows:

$$
\frac{\mathrm{CL}}{\mathrm{F}}=\mathrm{TV}_{1}(\mathrm{CL} / \mathrm{F}) \times \mathrm{OCC}+\mathrm{TV}_{2}(\mathrm{CL} / \mathrm{F}) \times(1-\mathrm{OCC})
$$

Where $\mathrm{TV}_{1}(\mathrm{CL} / \mathrm{F})$ is the mean apparent clearance for the first two days of treatment and $\mathrm{TV}_{2}(\mathrm{CL} / \mathrm{F})$ is the mean apparent clearance for the third day of treatment.
OCC(occasion) is equal to 1 for the first two days of treatment, and otherwise to 0 .

The significance of a relationship between a pharmacokinetic parameter and a covariate was assessed by use of the Chi-square test of the difference between the objective functions of the basic model (without the covariate) and the model with the covariate. A covariate was retained in the model if it produced a minimum decrease in the objective function of 3.64 units $(P=0.05,1$ degree of freedom) and if its effect was biologically plausible. An intermediate multivariate model that included all selected covariates was then obtained. A covariate was retained in the final multivariate model if its deletion from the intermediate model led to a 6.63-point increase in the objective function $(P=0.01,1$ degree of freedom). At each step, the goodness of fit was evaluated by use of a graph of the weighted residuals versus time after administration of the dose (time) or versus the predicted concentrations. Normalized predictions errors (NPDE) versus time and predicted concentrations were used to assess the lack of bias of the final model. NPDE are computed as the quantiles of the observations in the predicted distribution, obtained for each observation by simulating 1000 datasets using the model and the design of the original dataset. The computation also involves a decorrelation step to account for the correlation induced by the multiple observations within one subject. The distribution of the NPDE under the assumption that the model describes appropriately the observed data is the standard Gaussian distribution, and graphs of NDPE versus time and versus predicted concentrations can be used to evaluate this assumption [16].

The accuracy and robustness of the final population models were assessed by a visual predictive check. The final population model parameters were used to perform 1000 simulations of the database. The $5^{\text {th }}$ and the $95^{\text {th }}$ percentiles as well as the $50^{\text {th }}$ (median) of simulated concentrations were plotted against observed concentrations.

Bayesian estimates of the individual pharmacokinetic parameters (obtained in NONMEM output) were used to calculate individual MQ elimination half-life, the individual area under the curve from time zero to infinite (AUC), and MQ concentration at D7 (C7) and D28 (C28). Elimination half-life was calculated as follows: $\ln 2 / \beta$ with $\beta=0.5 \times$ $\left(\mathrm{Q} / \mathrm{Vc}+\mathrm{Q} / \mathrm{Vp}+\mathrm{CL} / \mathrm{Vc}-\left[(\mathrm{Q} / \mathrm{Vc}+\mathrm{Q} / \mathrm{Vp}+\mathrm{CL} / \mathrm{Vc})^{2}-4 \times\right.\right.$ $\mathrm{Q} / \mathrm{Vp} \times \mathrm{CL} / \mathrm{Vc}]^{1 / 2}$ ) and the calculation of $\mathrm{C} 7$ and $\mathrm{C} 28$ was made by NONMEM.

The primary efficacy outcome of the study was the PCR-uncorrected cure rate (proportion of patients with an adequate clinical and parasitological response) on D63 and PCR-corrected cure rate on D63 (proportion of patients without recrudescence/ inconclusive/ no results as classified by PCR genotyping). 
Table 1 Characteristics of the study population at the first drug intake

\begin{tabular}{|c|c|c|c|}
\hline & Mean & Standard deviation & Range \\
\hline Age (y) & 28.1 & 9.0 & $18-45$ \\
\hline Body weight (kg) & 53.3 & 7.3 & $40-70$ \\
\hline Parasite density (count/ $\mu \mathrm{L}$ ) & $8356^{*}$ & $N R$ & $1165-94693$ \\
\hline Haemoglobin (mg/dL) & 13.1 & 2.15 & 7.1-16.8 \\
\hline ASAT (IU) & 34.4 & 14.1 & $18-77$ \\
\hline ALAT (IU) & 26.2 & 17.1 & $1-85$ \\
\hline Serum creatinine & 0.96 & 0.18 & $0.6-1.7$ \\
\hline
\end{tabular}

*Median; NR: not relevant.

ASAT aspartate aminotransferase; ALAT alanine aminotransferase. ( $\mathrm{n}=77: 74$ male/3 female).

Comparison of MQ C7 and C28 between treatment success and treatment failure was expected to be performed by non-parametric statistical analyses.

\section{Results}

Seventy-seven patients (74 men, three women) were included. Their demographic and biological characteristics are given in Table 1. Three-hundred and fifty-five concentrations of MQ were available for the analysis. Distribution of sampling times can be seen on Figure 1.

The optimal model was a two-compartment model with first-order absorption and elimination. The estimated parameters were the absorption rate constant (ka), the apparent central distribution volume $(\mathrm{Vc} / \mathrm{F})$, the apparent peripheral distribution volume $(\mathrm{Vp} / \mathrm{F})$, the apparent elimination clearance (CL/F), and the apparent distribution clearance $(\mathrm{Q} / \mathrm{F})$, where $\mathrm{F}$ is the bioavailability. Inter-individual variability of $\mathrm{Q} / \mathrm{F}$ could not be estimated. Residual variability was described by a proportional error model.

BW was the only significant covariate and was found to explain the inter-individual variability of $\mathrm{Vp} / \mathrm{F}$ and $\mathrm{Vc} / \mathrm{F}$. All other covariates, including the day of dosing was not found to significantly influence the inter-individual variability of the PK parameters. The parameter estimates are given in Table 2.

Epsilon shrinkage was $19.8 \%$, eta shrinkage was $21 \%$ for CL/F, 34\% for $\mathrm{Vc} / \mathrm{F}, 53 \%$ for $\mathrm{Vp} / \mathrm{F}$, and $20 \%$ for $\mathrm{ka}$.

The lack of bias of the final model is observed in Figures 1 and 2. Visual predictive checks for the final model are displayed in Figure 3 and 11.4\% of the observed concentrations were outside the $90 \%$ confidence interval.

Median (range) mefloquine AUC was 1055 (596 - 1741 mg.h/l). Median (range) C7 was 1.58 (1.08 - 2.90) mg/l, and median (range) C28 was 0.29 (0.11 - 0.57) $\mathrm{mg} / \mathrm{l}$. Since no recrudescence and only one new infection was observed over the follow-up of the study (PCR-corrected cure rate of $100 \%$ ), no correlation between MQ C7 or C28 and treatment efficacy could be investigated.
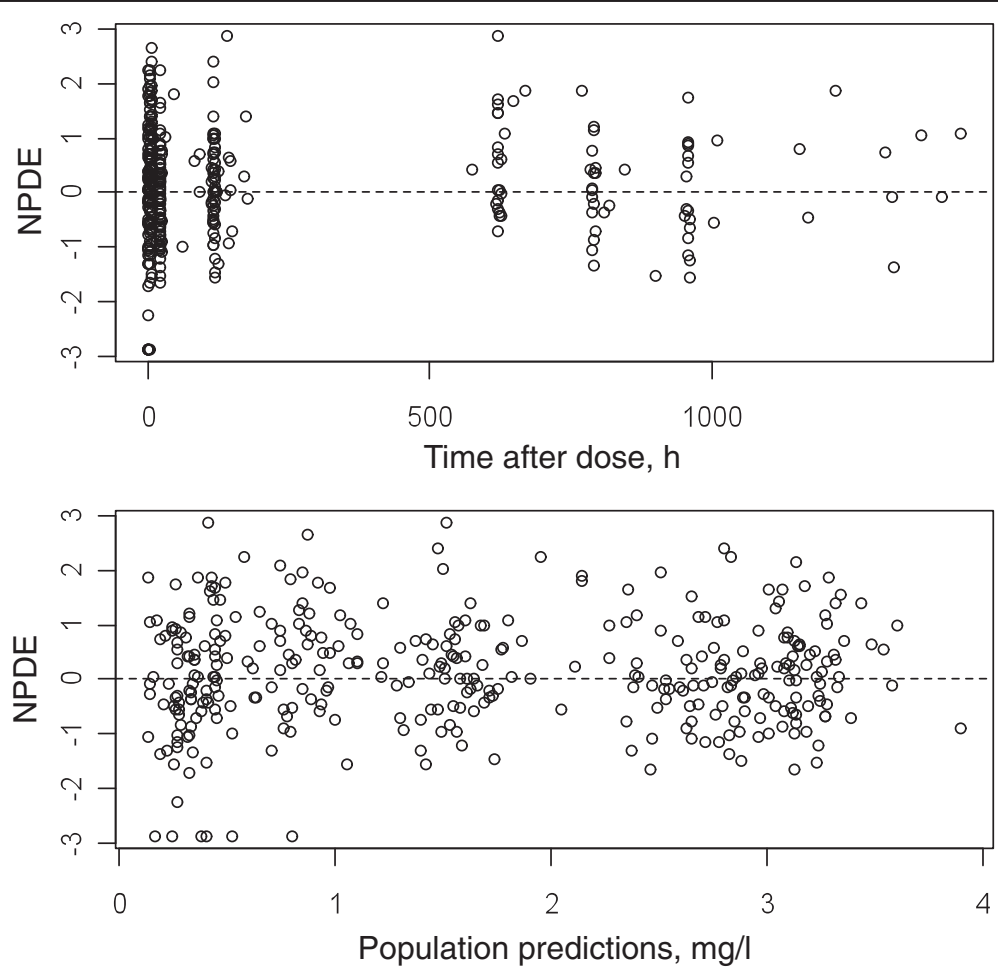

Figure 1 Lack of bias evidenced for the final model by the normalized predictions errors (NPDE) versus time after dose (T1), expressed in hours, and population predictions (PRED). 
Table 2 Parameter estimates

\begin{tabular}{|c|c|c|c|c|}
\hline \multirow[b]{2}{*}{ Parameter } & \multicolumn{2}{|c|}{ Base model } & \multicolumn{2}{|c|}{ Final model } \\
\hline & Estimate & SD & Estimate & SD \\
\hline $\mathrm{Ka}\left(\mathrm{h}^{-1}\right)$ & 0.166 & 0.0266 & 0.163 & 0.0263 \\
\hline$C L / F(L / h)$ & 1.15 & 0.0496 & 1.13 & 0.0501 \\
\hline $\mathrm{Vc} / \mathrm{F}(\mathrm{L})$ & 279 & 14.7 & 271 & 14.1 \\
\hline$\Theta_{B W, V C}$ & / & / & 0.87 & 0.288 \\
\hline $\mathrm{Q} / \mathrm{F}(\mathrm{L} / \mathrm{h})$ & 1.33 & 0.124 & 1.43 & 0.152 \\
\hline $\mathrm{Vp} / \mathrm{F}(\mathrm{L})$ & 341 & 39 & 344 & 40.6 \\
\hline$\Theta_{\mathrm{BW}, \mathrm{Vp}}$ & / & / & 2.41 & 1.08 \\
\hline$\omega_{k a}^{2}$ & 0.675 & 0.250 & 0.562 & 0.232 \\
\hline$\omega_{c l / F}^{2}$ & 0.0939 & 0.0224 & 0.0894 & 0.0218 \\
\hline$\omega_{v c / F}^{2}$ & 0.0603 & 0.0209 & 0.0453 & 0.0163 \\
\hline$\omega_{v_{p} / F}^{2}$ & 0.401 & 0.166 & 0.295 & 0.128 \\
\hline$\sigma^{2}$ & 0.0689 & 0.0150 & 0.0692 & 0.015 \\
\hline
\end{tabular}

$S D$ standard deviation of the estimate (obtained from the covariance step); Ka: absorption rate constant; $\mathrm{CL} / \mathrm{F}$ : apparent oral clearance; $\mathrm{V} / \mathrm{F}$ : apparent central distribution volume; $\Theta_{B W, v c}$ influential factor of $B W$ on $V c / F ; Q / F$ : apparent distribution clearance; $V p / F$ : apparent distribution volume $; \Theta_{\mathrm{BW}, \mathrm{V}_{\mathrm{p}}}$ : influential factor of $\mathrm{BW}$ on $\mathrm{Vp} / \mathrm{F} ; \omega_{\mathrm{CL} / \mathrm{s}}^{2}$ : inter-individual variability of $\mathrm{CL} / \mathrm{F} ; \omega_{\mathrm{VC} / /}^{2}$ : inter-individual variability of $\mathrm{Vc} / \mathrm{F} ; \omega_{v_{0} /:}^{2}$ : inter-individual variability of $\mathrm{Vp} / \mathrm{F} ; \omega_{k a}^{2}$ : inter-individual variability of $\mathrm{Ka} ; \sigma^{2}$ : proportional residual error.

\section{Discussion}

The present model accurately described the data and was in agreement with previous results seen in adult patients with uncomplicated malaria (Table 3), even though the present study was performed on plasma samples and most of these previous results were based on the concentration of MQ in whole blood. This can be

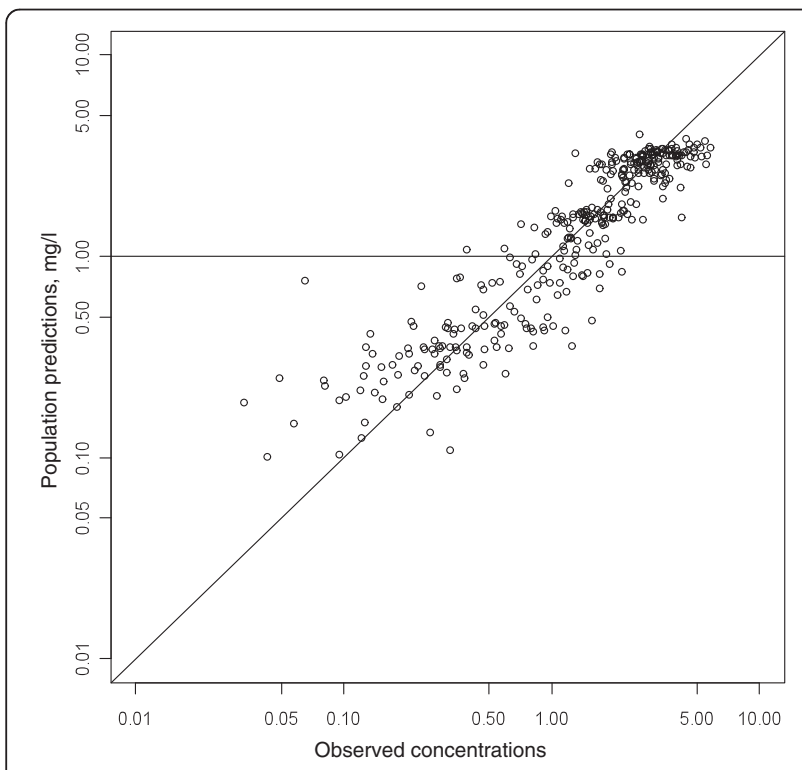

Figure 2 Population predictions versus observed mefloquine concentrations. Solid diagonal line: identity $(y=x)$ line.

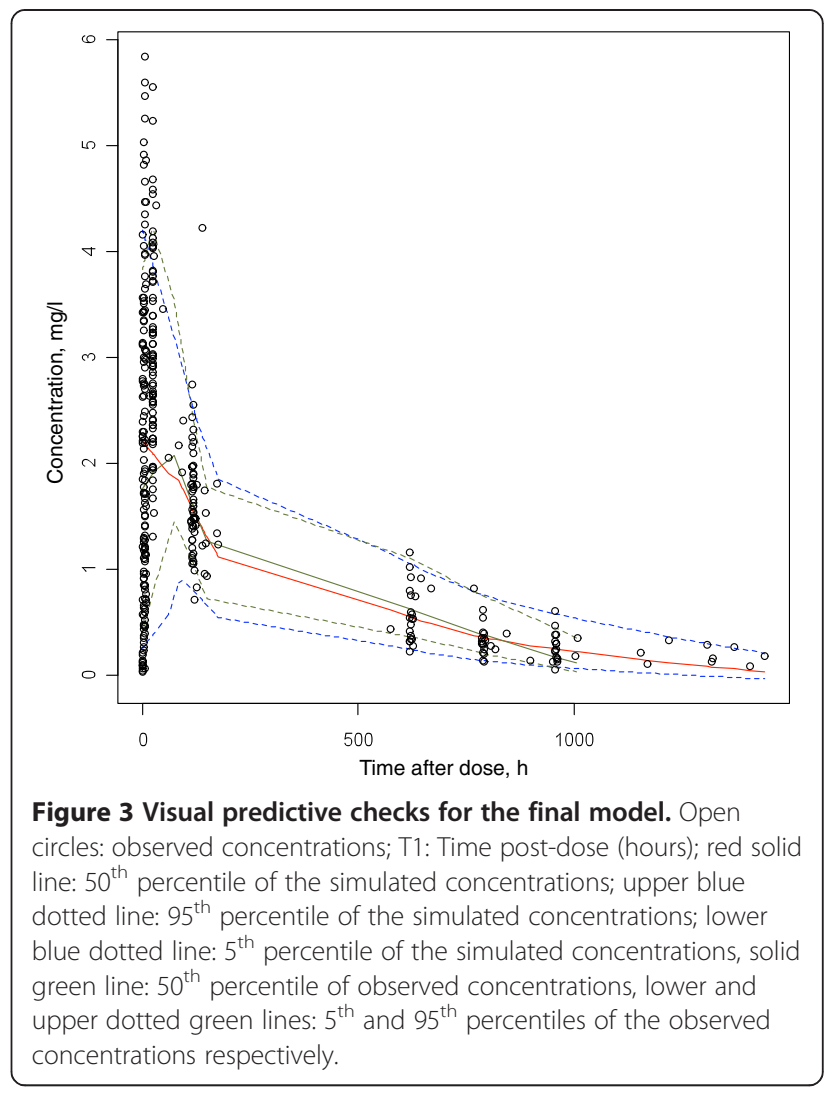

explained by the blood to plasma ratio of MQ which is close to 1 [17]. This consistency with results from studies performed in non-Indian patients suggests there are no ethnic-related differences in MQ pharmacokinetics, so a similar dosing regimen should achieve similar concentrations in Thai and in Indian patients. This result also confirms the bioavailability of the dosage form used in the present study (a FDC of AS and $\mathrm{MQ}$ ) is similar to the bioavailability of the non-fixed dose MQ forms that were used in previous studies described by Krudsood et al. [18]. A slightly longer elimination half-life was found in the present study, which may be due to differences in study design and in the sensitivity of the analytical methods. Indeed, delayed sampling times were available in the present study, and the quantification of MQ was possible in these samples thanks to the low LLOQ of the analytical method $(9.9 \mathrm{ng} / \mathrm{ml}$, compared to previous LLOQs of about $50 \mathrm{ng} / \mathrm{ml}$ (Krudsood, Simpson), $70 \mathrm{ng} / \mathrm{ml}$ (Ashley), $100 \mathrm{ng} / \mathrm{ml}$ (Charles), which allowed the determination of the two-compartment disposition model.

Differences in study design probably also explain why no modification in MQ pharmacokinetics during the treatment administration period could be detected. This change was previously described and attributed to the rapid clinical improvement seen due to treatment 
Table 3 Pharmacokinetics parameters of mefloquine in the present work and in previous studies

\begin{tabular}{|c|c|c|c|c|c|}
\hline Parameter & Gutman et al. [19] & Charles et al. [20] & Ashley et al. [21] & Krudsood et al. [18] & Current study \\
\hline Country of study & Peru & Australia & Thailand & Thai-Burmese border & India \\
\hline context & $\begin{array}{l}\text { uncomplicated } \\
\text { falciparum malaria }\end{array}$ & prophylaxis & $\begin{array}{l}\text { uncomplicated } \\
\text { falciparum malaria }\end{array}$ & $\begin{array}{l}\text { uncomplicated } \\
\text { falciparum malaria }\end{array}$ & $\begin{array}{l}\text { uncomplicated } \\
\text { falciparum malaria }\end{array}$ \\
\hline \multicolumn{6}{|c|}{ Age (yr): Mean (range) } \\
\hline & 36 & 26 & 19 (Median) & 27.8 & 28 \\
\hline & $18-61$ & $18-55$ & $2-55$ & $16-50$ & $18-45$ \\
\hline \multicolumn{6}{|l|}{ BW $(\mathrm{kg})$} \\
\hline Mean & NA & 82 & 44.5 (Median) & 51 & 53.3 \\
\hline Range & NA & $53-135$ & $10-63$ & $40-65$ & $40-70$ \\
\hline \multirow[t]{2}{*}{$\mathrm{CL} / \mathrm{F}$} & $0.017 \mathrm{~L} / \mathrm{h} / \mathrm{kg}$ & $2.09 \mathrm{~L} / \mathrm{h}$ & $1.33 \mathrm{~L} / \mathrm{h}$ & $0.024 \mathrm{~L} / \mathrm{h} / \mathrm{kg}$ & $1.13 \mathrm{~L} / \mathrm{h}$ \\
\hline & & & & & $0.022 \mathrm{~L} / \mathrm{h} / \mathrm{kg}$ \\
\hline \multirow[t]{2}{*}{$\mathrm{V} / \mathrm{F}$} & $8.57 \mathrm{~L} / \mathrm{kg}$ & $1011 \mathrm{~L}$ & $488 \mathrm{~L}$ & NA & $615 \mathrm{~L}$ \\
\hline & & & & & $11.9 \mathrm{~L} / \mathrm{kg}$ \\
\hline $\mathrm{T}_{1 / 2}$ & 14.5 days & 14 days & 10.5 days & 13.4 days & 21.6 days \\
\hline
\end{tabular}

NA not available.

efficacy [22]. There was indeed only one sample drawn within the first three days of treatment, which precluded the identification of early changes in MQ pharmacokinetics.

In agreement with previous findings [20], BW did not account for the inter-individual variability of $\mathrm{MQ} \mathrm{CL} / \mathrm{F}$, so lending support for the current use of a constant dose, independent of $\mathrm{BW}$, from one adult subject to another, consistent with previous population pharmacokinetics model performed in adults [20]. These results contrast with those of Ashley et al. [21], who reported increasing BW was associated with a reduction in MQ clearance, however this study included both children and adults so the relationship between clearance and BW may have been contributed by the paediatric population. Nevertheless, a significant relationship between $\mathrm{BW}$ and the central and peripheral distribution volumes was found, with a proportional increase in MQ elimination half-life with increasing BW observed (Figure 4). The prolonged presence of MQ in subjects with higher BW may have some impact on the prevention of reinfection, since it will take more time for MQ levels to fall below effective concentration levels [23]. However, the practical consequences of this theoretical finding should be further investigated. A similar increase in the clearance and distribution volume of $\mathrm{MQ}$, secondary to combination with artesunate, and leading to a decrease in MQ Cmax and AUC of about 25\%, was previously reported by Karbwang et al. [24]. The mechanism of this interaction could rely on a decrease in the bioavailability or in the protein binding of MQ. To our knowledge, this PK interaction was not further investigated. Anyway, because of the good efficacy of the AS/MQ combination, the clinical relevance of this interaction is unclear.

\section{Conclusions}

The present study provided population pharmacokinetics parameters for $\mathrm{MQ}$, administered as a FDC of $\mathrm{AS} / \mathrm{MQ}$, in Indian adult patients with acute uncomplicated $P$. falciparum malaria. The lack of relevant biological covariates on MQ CL/F, combined with the excellent efficacy results that were obtained, support the use of the 200/400 mg dose of AS/MQ in this population.

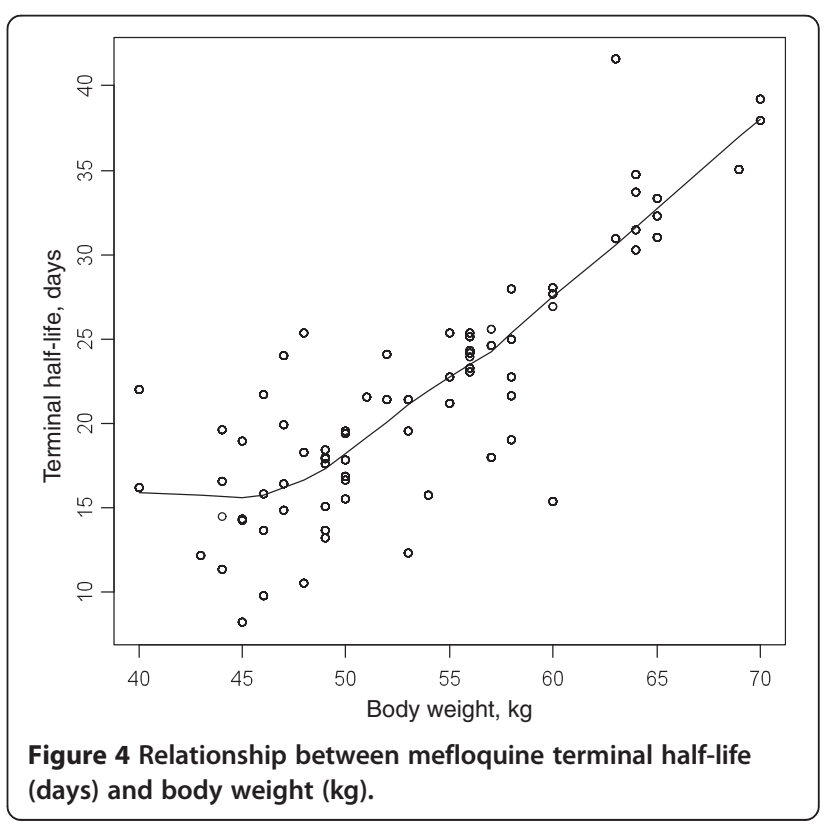




\section{Abbreviations}

ACT: Artemisinin combination therapy; AS: Artesunate; ASAT: Aspartate aminotransferase; ALAT: Alanine aminotransferase; BW: Body weight; DHA: Dihydroartemisinin; MQ: Mefloquine; NPDE: Normalized predictions errors; PRED: Population predictions.

\section{Competing interests}

The authors have declared that they have no competing interests.

\section{Authors' contributions}

VJ pharmacokinetic analysis, writing of the manuscript; BSh study coordination and monitoring; NV study management and execution; BSr technical overview; J-RK study pharmacokinetics protocol, contribution to the manuscript and discussion of the results. All authors read and approved the final manuscript.

\section{Acknowledgements}

Clinical trials were monitored by GVK (India), and the bio-analytical determinations were performed by Sitec Labs, Mumbai (India). We thank the following for their contribution in conducting the clinical study: N G Dubhashi (Goa Medical College and Hospital, Bambolim), B H Krishnamoorthy Rao (Wenlock District Government Hospital, Mangalore), Ashwani Kumar (National Institute of Malaria Research - Field Unit, Panjim), S K Ghosh (National Institute of Malaria Research, Bengaluru), Jai Prakash Narayan Singh and A P Dash (both of National Institute of Malaria Research, New Delhi). We thank patients for participating in the study and the local health personnel who contributed to the study execution, and acknowledge the assistance of Susan Wells (DNDI) in editing the manuscript. This manuscript bears the NIMR publication screening committee approval no. 01/2014. This work was supported by the Department for International Development, UK, the Dutch Ministry of Foreign Affairs, the Netherlands, and the French Development Agency, France.

\section{Author details}

${ }^{1}$ INSERM U1129, University Paris Descartes, Paris, France. ${ }^{2}$ Service de Pharmacologie, Hôpital Européen Georges Pompidou; Assistance Publique - Hôpitaux de Paris, Paris, France. ${ }^{3}$ National Institute of Malaria Research, New Delhi, India. ${ }^{4}$ Drugs for Neglected Diseases initiative, F-79 Green Park Main, New Delhi 110016, India. ${ }^{5}$ Drugs for Neglected Diseases initiative, 15 chemin Louis-Dunant, 1202 Geneva, Switzerland.

Received: 15 January 2014 Accepted: 27 April 2014

Published: 23 May 2014

\section{References}

1. German PI, Aweeka FT: Clinical pharmacology of artemisinin-based combination therapies. Clin Pharmacokinet 2008, 47:91-102.

2. Nyunt MM, Plowe CV: Pharmacologic advances in the global control and treatment of malaria: combination therapy and resistance. Clin Pharmacol Ther 2007, 82:601-605.

3. de Oliveira AM, Chavez J, de Leon GP, Durand S, Arrospide N, Roberts J, Cabezas C, Marquino W: Efficacy and effectiveness of mefloquine and artesunate combination therapy for uncomplicated Plasmodium falciparum malaria in the Peruvian Amazon. Am J Trop Med Hyg 2011, 85:573-578.

4. Mayxay M, Keomany S, Khanthavong M, Souvannasing P, Stepniewska K, Khomthilath T, Keola S, Pongvongsa T, Phompida S, Ubben D, Valecha N, White $\mathrm{NJ}$, Newton PN: A phase III, randomized, non-inferiority trial to assess the efficacy and safety of dihydroartemisinin-piperaquine in comparison with artesunate-mefloquine in patients with uncomplicated Plasmodium falciparum malaria in southern Laos. Am J Trop Med Hyg 2010, 83:1221-1229.

5. Faye B, Ndiaye JL, Tine R, Sylla K, Gueye A, Lo AC, Gaye O: A randomized trial of artesunate mefloquine versus artemether lumefantrine for the treatment of uncomplicated Plasmodium falciparum malaria in Senegalese children. Am J Trop Med Hyg 2010, 82:140-144.

6. WHO: WHO 19th Expert Committee on the Selection and use of Essential Medicines; 2012.

7. WHO: Guidelines for the Treatment of Malaria 2nd edition: 2010

8. Lacaze C, Kauss T, Kiechel JR, Caminiti A, Fawaz F, Terrassin L, Cuart S, Grislain L, Navaratnam V, Ghezzoul B, Gaudin K, White NJ, Olliaro P, Millet P: The initial pharmaceutical development of an artesunate/amodiaquine oral formulation for the treatment of malaria: a public-private partnership. Malar J 2011, 10:142
9. Ashley EA, Lwin KM, McGready R, Simon WH, Phaiphun L, Proux S, Wangseang N, Taylor W, Stepniewska K, Nawamaneerat W, Thwai KL, Barendo M, Leowattana W, Olliaro P, Singhavisanon P, White NJ, Nosten F: An open label randomized comparison of mefloquine- artesunate as separate tablets vs. a new co-formulated combination for the treatment of uncomplicated multidrug-resistant falciparum malaria in Thailand. Trop Med Int Health 2006, 11:1653-1660.

10. Smithuis F, Kyaw MK, Phe O, Win T, Aung PP, Oo AP, Naing AL, Nyo MY, Myint NZ, Imwong M, Ashley E, Lee SJ, White NJ: Effectiveness of five artemisinin combination regimens with or without primaquine in uncomplicated falciparum malaria: an open-label randomised trial. Lancet Infect Dis 2010, 10:673-681.

11. Leang R, Ros S, Duong S, Navaratnam V, Lim P, Ariey F, Kiechel J-R, Ménard D, Taylor WR: Therapeutic efficacy of fixed dose artesunate mefloquine for the treatment of acute, uncomplicated Plasmodium falciparum malaria in Kampong Speu. Cambodia. Malar J 2013, 12:343.

12. Santelli AC, Ribeiro I, Daher A, Boulos M, Marchesini PB, dos Santos RC, Lucena MBF, Magalhaes I, Leon AP, Junger W, Ladislau JL: Effect of artesunate-mefloquine fixed-dose combination in malaria transmission in amazon basin communities. Malar J 2012, 11:286.

13. Anvikar AR, Sharma B, Sharma SK, Ghosh SK, Bhatt RM, Kumar A, Mohanty SS, Pillai CR, Dash AP, Valecha N: In vitro assessment of drug resistance in Plasmodium falciparum in five States of India. Indian J Med Res 2012, 135:494-499.

14. Valecha N, Srivastava B, Dubhashi NG, Rao BHK, Kumar A, Ghosh SK, Singh JPN, Kiechel JR, Sharma B, Jullien V, Dash NG, Taylor WR, Anvikar AR: Safety, efficacy and population pharmacokinetics of fixed-dose combination of artesunatemefloquine in the treatment of acute uncomplicated Plasmodium falciparum malaria in India. J Vector Borne Dis 2013, 50:258-264.

15. Beal SL, Sheiner LB: NONMEM user's guide. San Francisco: NONMEM Project Group, University of California at San Francisco; 1991.

16. Brendel K, Comets E, Laffont C, Laveille C, Mentre F: Metrics for external model evaluation with an application to the population pharmacokinetics of gliclazide. Pharm Res 2006, 23:2036-2049.

17. Tajerzadeh $\mathrm{H}$, Cutler DJ: Blood to plasma ratio of mefloquine: interpretation and pharmacokinetic implications. Biopharm Drug Dispos 1993, 14:87-91.

18. Krudsood S, Looareesuwan S, Tangpukdee N, Wilairatana P, Phumratanaprapin W, Leowattana W, Chalermrut K, Ramanathan S, Navaratnam V, Olliaro P, Vaillant M, Kiechel JR, Taylor WR: New fixed-dose artesunate-mefloquine formulation against multidrug-resistant Plasmodium falciparum in adults: a comparative phase Ilb safety and pharmacokinetic study with standard-dose nonfixed artesunate plus mefloquine. Antimicrob Agents Chemother 2010, 54:3730-3737.

19. Gutman J, Green M, Durand S, Rojas OV, Ganguly B, Quezada WM, Utz GC, Slutsker L, Ruebush TK 2nd, Bacon DJ: Mefloquine pharmacokinetics and mefloquine-artesunate effectiveness in Peruvian patients with uncomplicated Plasmodium falciparum malaria. Malar J 2009, 8:58.

20. Charles BG, Blomgren A, Nasveld PE, Kitchener SJ, Jensen A, Gregory RM, Robertson B, Harris IE, Reid MP, Edstein MD: Population pharmacokinetics of mefloquine in military personnel for prophylaxis against malaria infection during field deployment. Eur J Clin Pharmacol 2007, 63:271-278.

21. Ashley EA, Stepniewska K, Lindegardh N, McGready R, Hutagalung R, Hae R, Singhasivanon P, White NJ, Nosten F: Population pharmacokinetic assessment of a new regimen of mefloquine used in combination treatment of uncomplicated falciparum malaria. Antimicrob Agents Chemother 2006, 50:2281-2285.

22. Simpson JA, Price R, ter Kuile F, Teja-Isavatharm P, Nosten F, Chongsuphajaisiddhi T, Looareesuwan S, Aarons L, White NJ: Population pharmacokinetics of mefloquine in patients with acute falciparum malaria. Clin Pharmacol Ther 1999, 66:472-484.

23. White NJ: Assessment of the pharmacodynamic properties of antimalarial drugs in vivo. Antimicrob Agents Chemother 1997, 41:1413-1422.

24. Karbwang J, Na Bangchang K, Thanarbul A, Back DJ, Bunnag D, Harinasuta $\mathrm{T}$ : Pharmacokinetics of mefloquine alone or in combination with artesunate. Bull World Health Org 1994, 72:83-87.

doi:10.1186/1475-2875-13-187

Cite this article as: Jullien et al:: Population pharmacokinetics of mefloquine, administered as a fixed-dose combination of artesunate-mefloquine in Indian patients for the treatment of acute uncomplicated Plasmodium falciparum malaria. Malaria Journal 2014 13:187. 